\title{
MANAGEMENT OF RESISTANT MYOFASCIAL PAIN IN TEMPOROMANDIBULAR JOINT DISORDERS WITH PHOTOBIOMODULATION
}

\author{
Hamada Abdel Hie Hamid Mahran*, Mohamed Mostafa Elshamaa** and Samir Ali Elborolsy**
}

\begin{abstract}
Background: The existence of temporomandibular joint pain and/or dysfunction in masticatory muscles is characterized in temporomandibular disorders (TMDs). Non-surgical treatment is the first step in the treatment of TMDs. Photobiomodulation therapy (PBMT) is, due to its impact on biological processes, especially inflammation, considered as an adjuvant treatment modality in TMD cases.
\end{abstract}

Aim: To evaluate the effectiveness of PBMT in treatment of resistant myofascial pain in patients with TMDs .

Material and Methods: A total of 60 patients aged from 17 to 49 years, with temporomandibular joint (TMJ) myofascial pain or TMJ arthralgia were involved in this study, patients examined clinically and orthopantomographic radiographs were taken for all the patients to rule out any gross anatomical deformity in relation to the TMJ. All patients treated by PBMT or low level laser therapy (LLLT). Pain intensity, maximum active and passive mouth opening, lateral jaw motions were assessed before and immediately after finishing treatment and after 3 months follow up.

Results: Statistically significant results were achieved in all study parameters

Conclusion: This study shows that PBMT effectively relieves pain and improves TMJ functional outcomes in patients with TMDs.

KEYWORDS: Photobiomodulation, Low-level laser therapy, Temporomandibular joint, Temporomandibular disorder

\section{INTRODUCTION}

The clinical complaints of the TMJ, masticatory muscles and related anatomical structures can be collectively called TMDs, and considered the most common cause of of non-odontogenic pain in the orofacial region. ${ }^{1}$

\footnotetext{
* Lecturer of Maxillofacial Surgery, General Surgery Department, Faculty of Medicine, Assiut university, Egypt.

** Lecturer, Oral and Maxillofacial Surgery Department, Faculty of Dentistry. Beni-Suef University
} 
About $33 \%$ of adult populations, at least has one symptom of TMDs as muscle pain, headache, joint sounds and limited mouth opening. TMDs can be diagnosed by clinical history, clinical examination, and appropriate radiological measures. ${ }^{2}$

From the criteria used to diagnose the TMDs is, Research Diagnostic Criteria for Temporomandibular Joint Disorders (RDC/TMD), it includes two axes for differentiation between the different types. Axis 1 involves myofascial pains, arthralgia, disc displacement, osteoarthritis, and arthrosis., ${ }^{3,4}$

The etiology of TMDs still unclear, it is thought that it is multifactorial, biomechanical, biopsychosocial, neuromuscular and biological factors. ${ }^{5}$ Therefore, the best treatment is multidisciplinary approach such as physical therapy modalities including manual therapy ${ }^{6}$, ultrasound ${ }^{7}$, electrothera$\mathrm{py}^{8}$, transcutaneous electric nerve stimulation ${ }^{9}$ and laser therapy. ${ }^{10}$

TMDs are considered to be a subgroup of musculoskeletal disorders, this may explain reports of the successful use of physical therapy in their treatment. PBMT is one modality of physical therapy, used for its anti-inflammatory, analgesic, and simulative effects. ${ }^{11}$ Although PBMT is a treatment method commonly used in physiotherapy of musculoskeletal disorders, there are few studies that deal with its utilization in treatment of TMD. ${ }^{12}$

Treatment should be based upon proper diagnosis, possible etiology, symptoms and signs. Treatment should begin with lines of treatment that decrease pain, recover functions and enable patients to regain their daily activities. As usual, in musculoskeletal disorders, TMD symptoms and signs could be selflimiting. Little is known about which symptom or sign is to worsen throughout the disorder course. However, early surgical interference or severe occlusal therapies should be avoided. ${ }^{13}$

Stabilization splints or occlusal therapy is considered the most commonly used line of treatment of temporomandibular myofascial pain syndrome ${ }^{14}$, but there is a debate questioning the role of occlusion in the pathogenesis of TMDs, so new therapeutic modalities have been incorporated into research. ${ }^{15}$

Recently, PBMT, previously known as LLLT has gained popularity as a method of treatment of painful muscular disorders. PBMT has analgesic, antiinflammatory, regenerative actions ${ }^{16}$ and increases lymphatic drainage so, it reduces tissue edema and these effects can appear in short time period. ${ }^{17}$

LLLT is classified as soft laser, which produces low level energy output, does not cause increase of skin temperature. Its main effect is based on light absorption mechanism. The wavelength of this soft laser ranges between 630 and $1300 \mathrm{~nm}$. It enhances tissues producing analgesic, antiinflammatory action through direct irradiation. It affects cellular respiratory chain in mitochondria leading to increased vascularization and formation of fibroblast. ${ }^{18}$

Mechanism of analgesic and anti-inflammatory effects of laser therapy still unclear, and probably explained by elevating the level of beta endorphin in the spinal fluid, also it enhances urinary excretion of glucocorticoids, which act as inhibitors of beta endorphin synthesis. It is thought that it increases the pressure pain threshold that occurs through electrolytic nerve fiber blocking mechanism, also it inhibits the release of acetylcholine and histamine, and it reduces synthesis of bradykinin. Bio stimulation effect could be due to metabolic activation, acceleration of mitochondrial cellular respiratory chain, enhancement of vascularization and fibroblast formation. ${ }^{19,20}$

At a depth of $1 \mathrm{~cm}$ under the skin surface the intensity of laser is reduced to $10 \%$ of its value. Meaning that a laser with power density of 100 $\mathrm{mW} / \mathrm{cm}^{2}$ at the skin surface, it will be $10 \mathrm{~mW} / \mathrm{cm}^{2}$ at $1 \mathrm{~cm}$ below skin surface, and it will be $1 \mathrm{~mW} / \mathrm{cm}^{2}$ at $2 \mathrm{~cm}$ below the skin surface. Meaning that LLLT is safe to be used in TMJ region. ${ }^{21}$ 
The aim of this study was to evaluate the effect of PBMT previously known as LLLT on patients with temporomandibular myofascial pain and limitation of mouth opening.

\section{MATERIALS AND METHODS}

This study was enrolled on patients who attended to Oral and Maxillofacial Surgery Department, Faculty of Dentistry, BeniSuef University from January 2017 to June 2020. A signed informed written consent was obtained after explanation of the study protocol. The inclusion criteria included Patients who have been diagnosed with TMD according to RDC/TMD Axis-I were included in the study and failed to respond to conservative treatments. They were not eligible for the study according to the following exclusion criteria: 1 . patients with evident internal derangement, 2. occlusal disturbances, 3. previous TMJ arthroscopic or surgical interference, 4. rheumatoid arthritis, 5. patients with missed data, 6. patients with chronic medical diseases, 7. Patients with a recent history of trauma, 8. cancer patients, 9. patients who refused to participate in the study or those who did not signed an informed consent. The study was approved by the Faculty of dentistry Beni-Suef University Research Ethics Committee (FDBSUREC) under the IORG \#: IORG0010018.

\section{Data Collection}

1. History taking. 2. Complete careful clinical examination extra and intraoral, to exclude occlusal disturbances, manifestations of internal derangement as clicking, detection of most tender points on lateral joint surface, temporalis, masseter muscles. 3. Pain measurement before treatment was carried out using a visual analog scale (VAS). The patients were asked to rate the pain from 0 to 10. Zero indicated "no pain" and 10 showed "the greatest possible pain". 4. Orthopantomogram (OPG) was routine to all patients to exclude TMJ arthritic changes.

\section{Ttreatment procedure}

The treatment was performed using a Biolase Epic X diode laser system (Biolase, 4 Cromwell, Irvine, CA 92618, USA) (Fig. 1). A 7 W laser beam with a $2.8 \mathrm{~cm}^{2}$ spot size emitting radiation continuously at a wavelength of $940 \mathrm{~nm}$, application was achieved every $48 \mathrm{~h}$ for 10 days, each application lasted 2 min (24 s per application point) so that each patient received approximately $300 \mathrm{~J}$ of energy per treatment. PBMT applied by the therapist extraorally to 5 points at the temporal (center of Temporalis muscle), zygomatic (origin of Masseter muscle), angle of the mandible (insertion of Masseter muscle), pre-auricular, and mastoid areas. Before starting this treatment patients instructed to stop the use of any occlusal splints or the intake of analgesic drugs. Patient position is semi setting 45 degrees on the dental unit, before laser application both treating physian and patient wear protection eye glasses to avoid eye injury.

The assessment of the following parameters was performed at three different points of time; before treatment, after the final treatment, and after 3 months follow up

1. Subjective pain intensity was accomplished by patient self assessment using (VAS)

2. Objective maximum active mouth opening, maximum passive mouth opening, Rt and Lt lateral jaw motion.



Fig. (1) Photobiomodulation dispensing system 
The maximum active mouth opening was measured by asking the patient to open his/her mouth as much as possible, then measuring the interincisal distance in mm. (Fig. 2) The maximum passive mouth opening was measured by application of downward pressure on the mandible by second and third fingers of the patient, then measuring the interincisal distance in $\mathrm{mm}$. The lateral jaw motion was measured as the horizontal distance between midpoints of upper and lower incisors in mm. (Fig. 3)

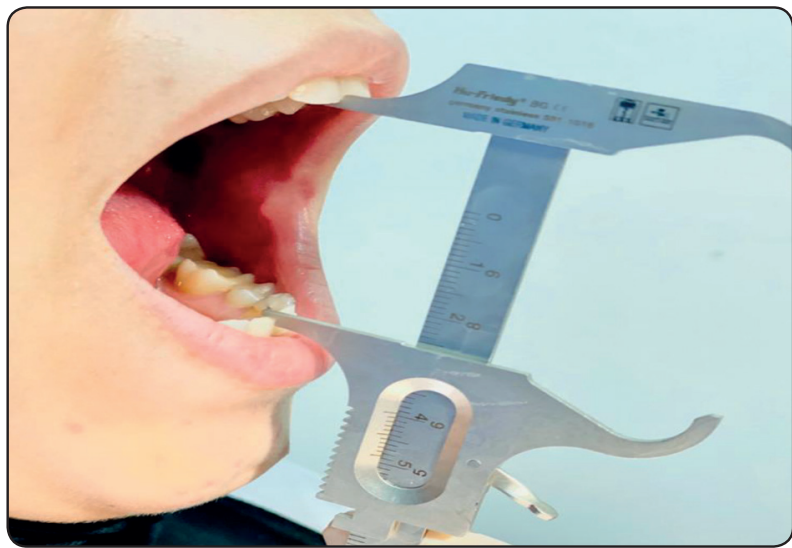

Fig. (2) Measurement of maximum active mouth opening



Fig. (3) Measurement of lateral jaw motion

\section{Statistical analysis of the data}

Data were fed to the computer and analyzed using IBM SPSS software package version 20.0. (Armonk, NY: IBM Corp). The Kolmogorov- Smirnov was used to verify the normality of distribution of variables. ANOVA with repeated measures was used for normally distributed quantitative variables, to compare between more than two periods and Post Hoc test (Bonferroni adjusted) for pairwise comparisons. Friedman testwas used for abnormally distributed quantitative variables, to compare between more than two periods and Post Hoc Test (Dunn's) for pairwise comparisons. The significance of the obtained results was judged at the $5 \%$ level.

\section{RESULTS}

Forty eight females and 12 males participated in this study who have been diagnosed with TMD according to RDC/TMD Axis I and failed to respond to other conservative treatments; the age range was from 17 to 49 . The presenting symptoms were formulated in (Table 1)

TABLE (1) Presenting symptoms

\begin{tabular}{ccc}
\hline Presenting symptom & $\begin{array}{c}\text { Number of } \\
\text { patients }\end{array}$ & Percentage \\
\hline Pain & 60 & $\mathbf{1 0 0 \%}$ \\
Limitation of mouth opening & 44 & $\mathbf{7 4 \%}$ \\
Deviation of mouth on opening & 39 & $\mathbf{6 5 \%}$ \\
\hline
\end{tabular}

Significant reduction in pain severity was observed by most patients and was maintained for three months, after completing of treatment plan; the Median (Min. - Max.) of the VAS before treatment sessions was $8(6-9)$ which significantly decreased to $0(0-5)$ and $0(0-4)$ immediately after treatment and 3 months follow up, respectively. (Table 2) Significant improvement seen in TMJ functional aspects as, maximum active and passive mouth opening and lateral jaw motions. The mean of the maximum active mouth opening before treatment sessions was $29.2 \pm 3.9 \mathrm{~mm}$, and there was a significant improvement in the measurements of the maximum active mouth opening up to 12 $\mathrm{mm}$ in each patient. The mean of the maximum active mouth opening, after treatment sessions, was $41.9 \pm 2.5 \mathrm{~mm}$ and $41.7 \pm 2.3 \mathrm{~mm}$ at the end of the 
follow-up period with significant statistical results. Regarding the mean of the maximum passive mouth opening, it was $9.82 \pm$, and significantly increased to $19 \pm 2.1$ immediately after finishing treatment sessions. After 3 months, it significantly decreased to $18.62 \pm$. The mean of the Rt lateral jaw motion and Lt lateral jaw motion before treatment was 4.9 $\pm 1.1 \mathrm{~mm}$ and $4.7 \pm 1.1$ respectively which was also significantly improved to be $8.10 .9 \pm \mathrm{mm}$ and $8.5 \pm$ 1 after treatment, respectively. (Table. 2)

\section{DISCUSSION}

TMD remains to be a complex disorder which is still difficult to define, and could be challenging in proper diagnosis and treatment. Constantly physical therapy continues to play a major role in its conservative management..$^{22}$ In the recent years, low-level laser therapy is used successfully to relieve TMD pain and improve TMJ functions. ${ }^{23}$

In this study we found that TMD is most dominant among females, $80 \%$ of the patients were

TABLE (2): Comparison between the three periods according to the different parameters $(n=60)$

\begin{tabular}{|c|c|c|c|c|}
\hline & Pre & Post & 3month & $\mathbf{p}$ \\
\hline \multicolumn{5}{|l|}{ Pain intensity } \\
\hline Mean \pm SD & $8 \pm 0.8$ & $0.5 \pm 1.1$ & $0.9 \pm 1.3$ & $<0.001^{*}$ \\
\hline \multirow{2}{*}{ Median (Min. - Max.) } & $8(6-9)$ & $0(0-5)$ & $0(0-4)$ & \\
\hline & $\mathrm{p}_{1}<0.001^{*}$ & $\mathrm{p}_{2}<0.001^{*}$ & $\mathrm{p}_{3}=0.273$ & \\
\hline \multicolumn{5}{|c|}{ Maximum active mouth opening } \\
\hline Mean \pm SD & $29.2 \pm 3.9$ & $41.9 \pm 2.5$ & $41.7 \pm 2.3$ & $<0.001^{*}$ \\
\hline \multirow{2}{*}{ Median (Min. - Max.) } & $29(22-41)$ & $42(35-47)$ & $42(34-46)$ & \\
\hline & $\mathrm{p}_{1}<0.001^{*}$ & $\mathrm{p}_{2}<0.001^{*}$ & $\mathrm{p}_{3}=0.341$ & \\
\hline \multicolumn{5}{|c|}{ Maximum passive mouth opening } \\
\hline Mean \pm SD & $9.8 \pm 2$ & $19 \pm 2.1$ & $18.6 \pm 2$ & $<0.001^{*}$ \\
\hline \multirow[t]{2}{*}{ Median (Min. - Max.) } & $9(7-16)$ & $19(14-23)$ & $18.5(13-23)$ & \\
\hline & $\mathrm{p}_{1}<0.001^{*}$ & $\mathrm{p}_{2}<0.001^{*}$ & $\mathrm{p}_{3}=0.003^{*}$ & \\
\hline \multicolumn{5}{|l|}{ Rt lateral jaw motion } \\
\hline Mean \pm SD & $4.9 \pm 1.1$ & $8.1 \pm 0.9$ & $8.4 \pm 0.9$ & $<0.001^{*}$ \\
\hline \multirow[t]{2}{*}{ Median (Min. - Max.) } & $5(3-8)$ & $8(5-10)$ & $8(7-10)$ & \\
\hline & $\mathrm{p}_{1}<0.001^{*}$ & $\mathrm{p}_{2}<0.001^{*}$ & $\mathrm{p}_{3}=0.002^{*}$ & \\
\hline \multicolumn{5}{|l|}{ Lt lateral jaw motion } \\
\hline Mean \pm SD & $4.7 \pm 1.1$ & $8.5 \pm 1$ & $8.1 \pm 1$ & $<0.001^{*}$ \\
\hline \multirow[t]{2}{*}{ Median (Min. - Max.) } & $5(3-7)$ & $9(6-10)$ & $8(6-10)$ & \\
\hline & $\mathrm{p}_{1}<0.001^{*}$ & $\mathrm{p}_{2}<0.001^{*}$ & $\mathrm{p}_{3}=0.014^{*}$ & \\
\hline
\end{tabular}

SD: Standard deviation

Fr: Friedman test, Sig. bet. Periods was done using Post Hoc Test (Dunn's)

F: F test (ANOVA) with repeated measures, Sig. bet. Periods was done using Post Hoc Test(adjusted Bonferroni) p: $p$ value for comparing between the studied periods p1:p value for comparing between Pre and Post p2:p value for comparing between Pre and 3month p3:p value for comparing between Post and 3month

*: Statistically significant at $p \leq 0.05$ 
females and female patients ask for medical advice more than males, this is similar to multiple other studies $^{24}$, this was also mentioned by Murrieta et al, ${ }^{25}$ who attributed this predominance in females due to emotional status, morphological characteristic and hormonal factors.

In the literature, still no consensus on frequency of PBMT, also no consensus on number of sessions, dose of laser applications. Some authors advocated eight sessions with two sessions per week ${ }^{26}$, other authors advocated six sessions with two sessions per week and have recommended applying the laser wand to areas with pain only. ${ }^{27}$ Furthermore, there is no consensus on the application points of the laser. We applied the laser wand to pre-established points for all the patients and parameters of the laser treatment are outlined in (Table 3 ).

The end results of the study are encouraging as regard the effectiveness of PBMT in the treatment of myofascial pains, there was marked improvement in subjective parameters such as pain, as well as in objective parameters such as maximum mouth opening, and lateral jaw motions. Improvement of joint functional aspects can be due to analgesic, bio stimulating effects of laser application.

Our results were the same as observed by Pala$\mathrm{no}^{28}$, who found that PBMT has therapeutic effect on myofascial pains, TMJ clicking, mouth opening and TMJ functional improvement and he explained that these findings could be due to the anti-inflammatory effect of laser therapy. Also Bezzur et al ${ }^{29}$ found that PBMT had therapeutic effect on the TMJ amplitude of movements and they explained this occurs as a result of anti-inflammatory effect of laser and due to alteration of secondary muscle inhibition that happens due to hyperactive sensory receptors in the joint.

In contrast with other authors as, De Aberu Venancio et al ${ }^{30}$, Chen etal ${ }^{31}$, who advocated that application of PBMT, has transient or no effect on TMJ pain relief.
These widely different results found in the literature could be explained by the variation in the therapy regimes, the wavelength, the pulse frequency, power output, and energy dosages of the laser used, also the variation in the sites of application and number of application points, all these variations make comparison so difficult. ${ }^{32}$

PBMT is a simple, safe, non-invasive, time saving, on pharmaceutical, well tolerated procedure, it has few or no side effects, it contributes to increased patient comfort. ${ }^{33,34} \mathrm{We}$ did not meet any adverse reactions in this study. PBMT has therapeutic effects on subjective and objective manifestations of temporomandibular myofascial syndrome with no reported complications.

Bigger sample studies, double blind random studies with control groups are indicated to accurately determine the effectiveness of laser therapy in treatment of TMDs, also to standardize the treatments protocol.

\section{REFERENCES}

1. De Leeuw R. In: The American Academy of Orofacial Pain, editor, Orofacial: guidelines for assessment, diagnosis, and management. 4thed. Quintessence :2008. P. 129-204.

2. Hanci M, Karamese M. Tosun Z, et al. Intra-articular platelet -rich plasma injection for the treatment of temporomandibular disorders and a comparison with arthrocentesis. J CraniomaxillofacSurg 2015 ;43:162-6.

3. Ahmad M, Hollender L, Anderson Q, et al. Research diagnostic criteria for temporomandibular disorders (RDC/TMD): development of image analysis criteria and examiner reliability for image analysis. Oral Surg Oral Med Oral Pathol Oral RadiolEndod 2009;107:844-60.

4. Talaat WM, Adel OI, AI Bayatti S. Prevalence of temporomandibular disorders discovered incidentally during routine dental examination using the Research Diagnostic Criteria for Temporomandibular Disorders. Oral Surg Oral Med Oral Pathol Oral RadiolEndod 2018;125:250-9.

5. Maria C,A., Monica P,A., Sever P.,et al. Factors involved in the etiology of temporomandibular disorders - a literature review. 2015;88(4):473-478. 
6. Albertin A., Kerppers I. I., Amorim C. F., Costa R. V., Ferrari Correa J. C., Oliveira C. S. The effect of manual therapy on masseter muscle pain and spasm. $2010 ; 50: 107-112$.

7. Zhou F. H., Zhao H. Y. Acupuncture and ultrasound therapy for temporomandibular disorders. 2004; 24: p. 720.

8. Gomes N. C., Berni-Schwarzenbeck K. C., Packer A. C., Rdrigues-Bigaton D. Effect of cathodal high-voltage electrical stimulation on pain in women with TMD. 2012; 16(1):10-15.

9. Rodrigues D., Siriani A. O., Berzin F. Effect of conventional TENS on pain and electromyographic activity of masticatory muscles in TMD patients. 2004;18(4):290295.

10. Ferreira L. A., de Oliveira R. G., GuimaraesJ. P., Carvalho A. C., De Paula M. V. Laser acupuncture in patients with temporomandibular dysfunction : a randomized controlled trial. $2013 ; 28(6)$ : 1549-1558.

11. Cetiner S,, Kahraman S,A., and Yucetas S, (2006). Evaluation Low-Level Laser therapy in the treatment of temporomandibulardisorders, Photomed. Laser Surg. 24, 637-641.

12. Medlicott, M,S., and Harris, S,R. (2006). A systematic review of the effectiveness of exercise, manual therapy, electrotherapy, relaxation training, and biofeedback in the management of temporomandibular disorder. Phys, Ther, 86, 955-973.

13. Venancio RA, Camparis CM, Lizarelli RFZ: Low intensity laser therapy in the treatment of temporomandibular disorders : a double blind study. J Oral Rehabil 2005; 32 : 800-807.

14. Ekberg E, Vallon D, Nilner M. The efficacy of appliance therapy in patients with temporomandibular disorders of mainly myogenous origin. A randomized, controlled, short-term trial. J Orofac Pain 2003;17: 133-9.

15. Chinsnoiu AM, Picos AM, Popa S, et al. Factors involved in the etiology temporomandibular disorders - a literature review. ClujulMed 2015; 88:473-548.

16. Maia MI., Bongardim LR., Quintans J de S, Riberio MA., Maia LG., Conti PC. Effect of low-level laser therapy on pain levels in patients with temporomandibular disorders : a systemic review. J Appl Oral Sci. 2012;20(6):594-602.

17. Cavalho CM, Lacerda JA, dos Santos Neto FP, et al. Evaluation of laser phototherapy in the inflammatory process of the rats TMJ induced by carrageenan. Photo Laser Surg 2011;29(4):245-254.
18. Skinner SM, Gage JP, Wilee PA et al. A preliminary study of the effects of laser radiation on collagen metabolism in cell culture. Aust Dent J 1996 41: 188-192.

19. Simunovic Z, Low-level laser therapy with trigger points technique : a clinical study on 243 patients. J Clin Laser Med Surg 1996;14 :163-167.

20. Simunovic Z. Pain and practical aspects of its management. In: Laser Medico, ed. Lasers in medicine and dentistry. Basic science and up-to-date clinical application of low energy level laser therapy - LLLT. Rijeka : European Medical Laser Association.2000; cap. 14:269-301.

21. M. Melis, M. Di Giosia, and K. H. Zawawi, Low level laser therapy for the treatment of temporomandibular disorders : a systemic review of the literature, The Journal of Craniomandibular Practice, vol, 30, no. 4,pp. 304-312,2012.

22. Girdler NM. In vitro synthesis and characterization of a cartilaginous meniscus grown from isolated temporomandibular chond ro progenitor cells. Scand J Rheumatol $1998 ; 27: 446-53$.

23. J. D. Carrol, M. R. Milward, P. R. Cooper, M.Hadis, and W. M. Palin, "Developments in low level light therapy (LLLT) for dentistry “, Dental material, vol. 30, no. 5, pp. 465-475, 2014.

24. Merrill, R. L. Manual of Temporomandibular Disorders, Second Edition. J. Orofac. Pain 2010;24: 210.

25. Jose Francisco Murrieta, Emma Laura Alvarado, Manuel Tomas Valdez, Leticia Orozco, Julieta del Carmen Meza, M. L. J. Prevalence of temporomandibularjoint disorders in a Mexican elderly group. J. Oral Res. 2016;5(1), 13-18.

26. M. L.D. M. Maia, L. R. Bonjardim, J. D. S. S. Quintans, M. A. G. Ribeiro, L. G. M. Maia, and P. C. R. Conti, "effect of low level laser therapy on pain levels in patients with temporomandibular disorders : a systematic review," Journal of Applied Oral Science, vol. 20,no. 6, pp. 594-602, 2012.

27. A. M. Shirani, N. Gutknecht, M. Taghizadeh, and M. Mir, "Low level laser therapy and myofacial dysfunction syndrome : a randomized controlled clinical trial," Lasers in Medical Science, vol. 24, no.5,pp. 715-720, 2009.

28. Palano D, Martelli M, Avi R, Gaurneri L, Palmieri B. A clinic stastistical investigation of laser effect in the treatment of pain and dysfuntionottemporomandibular joint (tmj). Med Laser Rep 1985;2:21-29.

29. Bezzur NJ, Habets LL, Hanson TL. The effect of therapeutic laser treatment in patients with craniomandibular disorders. J CraniomandibDisord 1988 2: 83-86. 
30. R. De Abreu Venancio, C. M. Camparis, and R. De Fatima ZaniratoLizarelli, "Low intensity laser therapy in the treatment of temporomandibular disorders : a double blind study, “ Journal of Oral Rehabilitation, vol. 32, no. 11, pp. 800-807, 2005.

31. Chen J., Huang Z., Ge M., Gao M. Efficacy of low-level laser therapy in the treatment of TMDs: a meta-analysis of 14 randomised controlled trials. 2015 ;42(4):291-299.

32. Mester E, Toth N, Mester A. The bioestimulative effect of laser beam, Lase Basic Biomed Res 1982 22: 4-7.

33. Carli M. L., Guerra M. B., Nunes T. B., et al. Piroxicam and laser phototherapy in the treatment of TMJ arthralgia :a double blind randomized controlled trial.2013; 40(3):171 -178.

34. Sancaki E., Gokeen -Rohling B., Balik A., Ongul., Kipirdi S., Keskin H. Early results of low-level laser application for masticatory muscle pain : a double-blind randomized clinical study. $2015 ; 15(1)$ :p. 131. 